\title{
Hemoglobin Detection on a Microfluidic Sensor Chip with a Partially Conjugated Polymer
}

\author{
Soo-Han Eo, Kwang-Jae Won, Simon Song, ${ }^{*}$ Bora Yoon, ${ }^{\dagger}$ and Jong-Man Kim ${ }^{\dagger}{ }^{*}$ \\ Department of Mechanical Engineering, Hanyang University, Seoul 133-791, Korea. *E-mail: simonsong@hanyang.ac.kr \\ ${ }_{\dagger}^{\dagger}$ Department of Chemical Engineering, Hanyang University, Seoul 133-791, Korea. *E-mail: jmk@hanyang.ac.kr \\ Received October 6, 2009, Accepted December 22, 2009
}

Key Words: Conjugated polymer, Sensor, Fluorescence, Hemoglobin, Microfluidic chip

The development of efficient chemosensors based on the conjugated polymers has been the central focus of a large number of recent research programs. ${ }^{1-18}$ The presence of extensively delocalized electrons and conformational restrictions of the backbone structures make conjugated polymers attractive sensory materials. In these polymers, molecular recognition events influence electronic absorption and emission properties. Thus, a wide variety of conjugated polymer-based sensors have been investigated. However, the majority of the conjugated polymer sensors described to date have been explored in the form of solutions or thin films.

Unlike conventional batch-type solution or film-based sensors, microfluidic detection systems provide several unique features including minimal quantities of samples and reagents, large interfacial areas, relatively fast molecular diffusion, and the capability of continuous analysis. ${ }^{19-22}$ Despite these attractive properties, the application of the conjugated polymers to microfluidic sensor systems has been exceptionally rare. ${ }^{23,24}$ In this note, we describe a microfluidic sensor system based on a novel water-soluble conjugated polymer.

Most biologically interesting target molecules, such as proteins, carbohydrates, nucleic acids, or ions, are only soluble in water. Thus, it is desirable to use water-soluble conjugated polymers as sensor matrices. In general, in order to make watersoluble conjugated polymers tedious procedures are required since most synthetic methods developed for this purpose are incompatible with sidechain functionalities. ${ }^{25}$ Accordingly, protecting group strategies are required to prepare polymers with requisite functional groups that foster water solubility.

The poly(phenylene-vinylene) (PPV) precursor, $\operatorname{poly}(p$ xylene tetrahydrothiophenium chloride) $\mathbf{1}$ is commercially available (Scheme 1). The precursor polymer 1 has been known to undergo elimination reaction to give the fully conjugated PPV 2 upon heat or base treatment. We felt that if the precursor

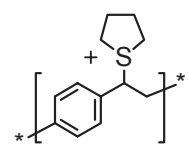

PPV Precursor

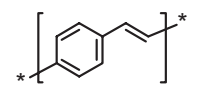

PPV

2

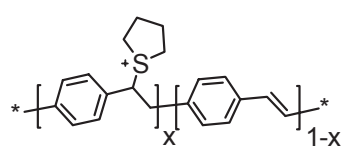

Partially Conjugated Polymer 3
Scheme 1. Heat-induced transformation of the PPV precursor to the partially conjugated polymer polymer 1 can be existed as a partially conjugated form such as $\mathbf{3}$, the conjugated polymer $\mathbf{3}$ would be soluble in aqueous solution. In fact, we found that the PPV precursor 1 was converted to a partially-conjugated polymer $\mathbf{3}$ when the polymer $\mathbf{1}$ was arrived due to the instability of the polymer 1 . Conversion of the precursor polymer $\mathbf{1}$ to the partially conjugated polymer 3 was confirmed by observing absorption maximum of the aqueous solution containing the PPV precursor 1 at $323 \mathrm{~nm}$. The PPV precursor polymer 1 should not absorb light above $300 \mathrm{~nm}$ since it contains only nonconjugated phenyl moieties. In order to gain more information on the partially conjugated polymer $\mathbf{3}$, the polymer solution was lyophilized to give a solid residue. The solid residue, however, was not soluble in any solvent including water, DMSO, DMF, acetone, THF, and chloroform. Thus, we were unable to obtain NMR spectroscopic data. We believe that the polymer was converted to the fully conjugated form of PPV 3 during lyophilization. The color of the solid reside obtained by lyophilization was yellow-green and the solid reside emits strong fluorescence, typical characteristics of the fully conjugated PPV 3. Thus, we decided to use the water soluble polymer $\mathbf{3}$ as received. Since hemoglobin is an excellent fluorescence quencher of conjugated polymers, ${ }^{26}$ we expected that the fluorescence of the polymer 3 would be quenched by interaction with this protein in a microfluidic channel and, as a result, the system would function as a hemoglobinsensitive conjugated polymer sensor chip.

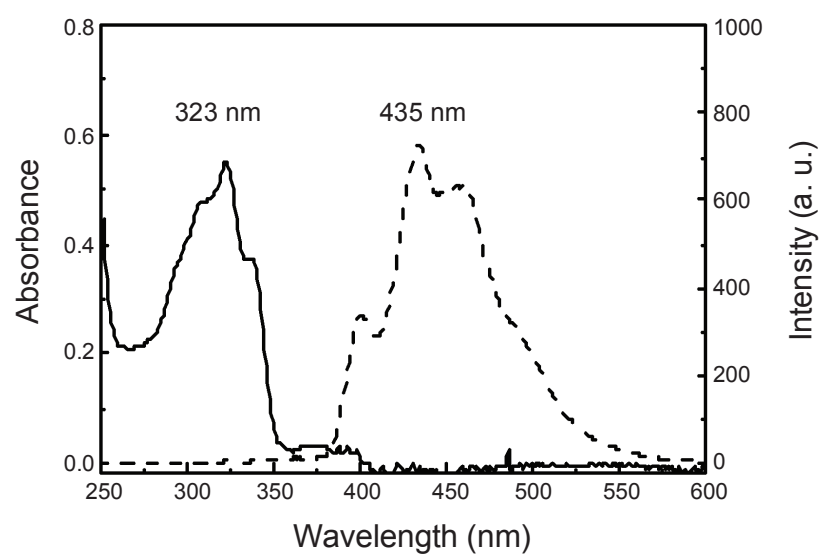

Figure 1. UV (solid line) and emission (dash line) spectra of an aqueous solution ( $c a .6 .5 \mathrm{mM}$ based on the monomer unit) containing the polymer 3 . 


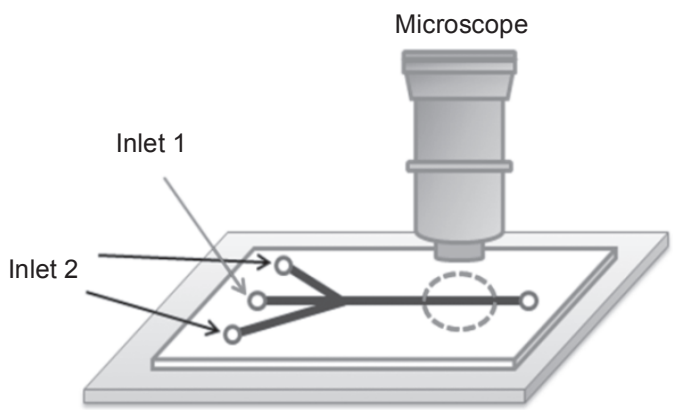

Figure 2. A schematic of the microfluidic sensor chip investigated in current study.
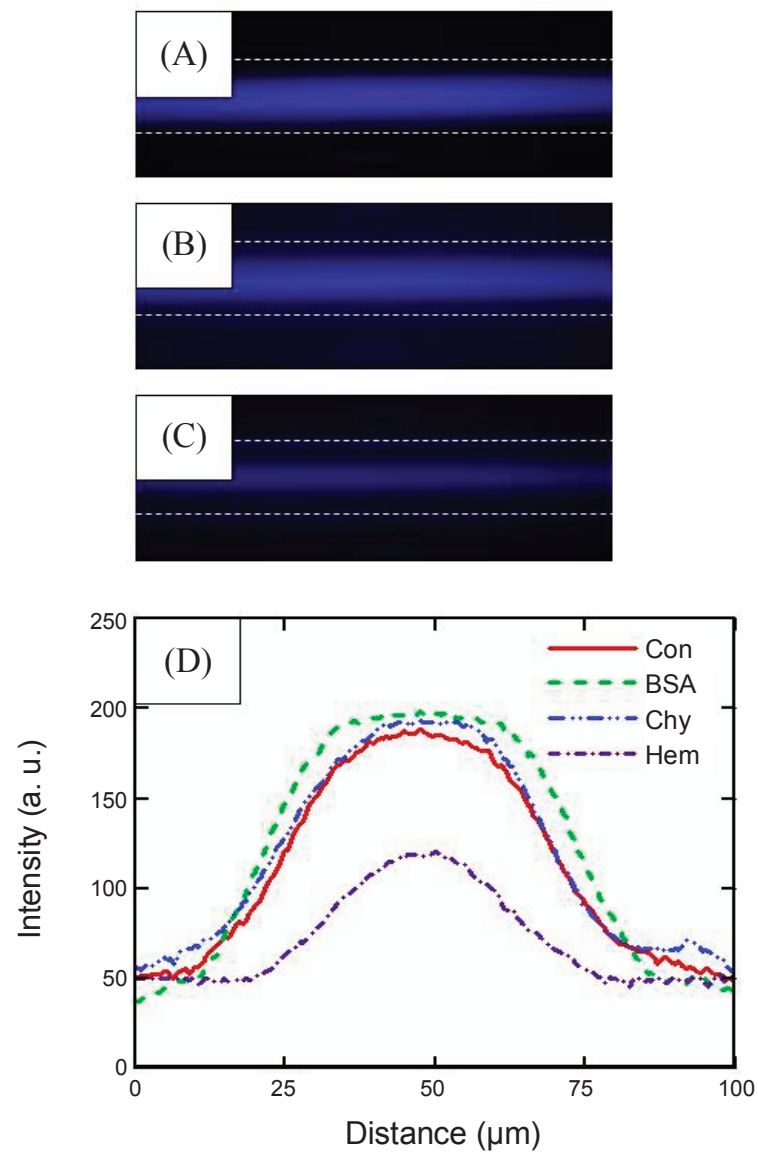

Figure 3. Fluorescence images (A-C) of microfluidic sensor chips upon interaction with $10 \mathrm{mg} / \mathrm{mL}$ BSA (A), $10 \mathrm{mg} / \mathrm{mL}$ chymotripsin (B), and $0.1 \mathrm{mg} / \mathrm{mL}$ hemoglobin $(\mathrm{C})$, respectively. Figure $3 \mathrm{D}$ contains fluorescent intensity profiles across the microchannel. Abbreviation: Con (control), BSA (bovine serum albumin), Chy (chymotripsin), and Hem (hemoglobin). All measurements were carried out in HEPES buffer solution ( $5 \mathrm{mM}, \mathrm{pH} 8.0$ ).

Figure 1 displays UV absorption and emission spectra of an aqueous solution (HEPES buffer, $5 \mathrm{mM}, \mathrm{pH} \mathrm{8.0)}$ ) containing the partially conjugated polymer 3 . The polymer solution is relatively strongly fluorescent due to the presence of conjugated phenylenevinylene moieties and has a maximum emission wavelength at $435 \mathrm{~nm}$.

A microfluidic sensor chip using hydrodynamic focusing (Figure 2) was fabricated by a standard soft lithographic method

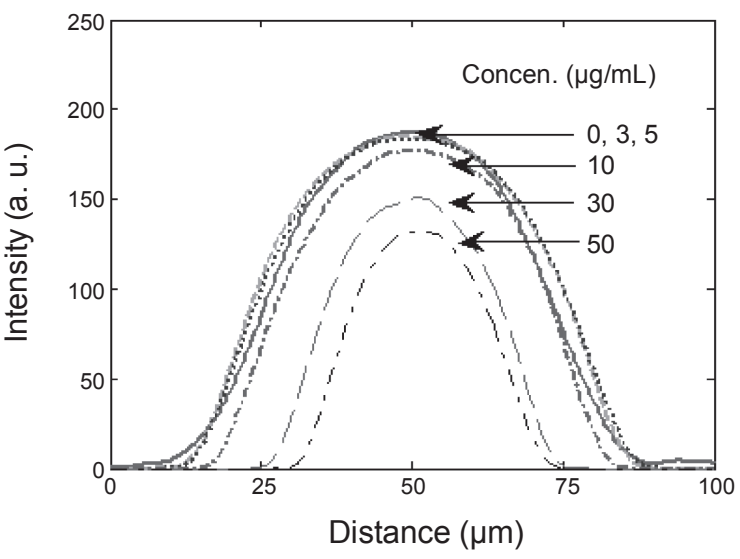

Figure 4. Fluorescence intensity profiles of the conjugated polymer 3 in the presence of various concentrations of hemoglobin (HEPES buffer, $5 \mathrm{mM}$, $\mathrm{pH} 8.0$ ).

described in our previous report. ${ }^{23} \mathrm{~A}$ stream of the polymer solution is introduced into the middle of microchannels (inlet 1) and is focused by sheath flows consisting of protein solutions (inlet 2). The flow rates of the conjugated polymer and protein solutions, controlled by syringe pumps connected to the inlets, are $0.03 \mathrm{~mL} / \mathrm{h}$ and $0.01 \mathrm{~mL} / \mathrm{h}$. The main microchannel dimensions are $100 \mu \mathrm{m}$ in width and $50 \mu \mathrm{m}$ in depth. Fluorescence microscope monitoring was carried out with a filtered (510 $530 \mathrm{~nm}$ ) excitation.

Figure 3 contains the fluorescence images obtained when a $100 \mu \mathrm{M}$ conjugated polymer flow is in contact with bovine serum albumin (BSA) (Figure 3A), chymotripsin (Figure 3B), and hemoglobin (Figure 3C). It is clear from inspecting these fluorescence images that hemoglobin is superior to the other protein in terms of its fluorescence quenching ability, even when a much lower concentration is used $(0.1 \mathrm{mg} / \mathrm{mL}$ of hemoglobin vs. $10 \mathrm{mg} / \mathrm{mL}$ of BSA and chymotripsin). A much narrower fluorescence band is observed when the polymer $\mathbf{3}$ contacts hemoglobin. The fluorescence intensity profiles shown in Figure 3D further demonstrate the fluorescence quenching ability of hemoglobin. The fluorescence intensity of polymer $\mathbf{3}$ is substantially decreased even when a 100 times lower concentration of hemoglobin is used. The fluorescent quenching of the conjugated polymer by hemoglobin is believed to occur by singlet energy transfer from the conjugated polymer to the heme group of the protein. ${ }^{26}$

We next investigated the detection limit of hemoglobin by using the polymer $\mathbf{3}$ based microfluidic sensor chip. In Figure 4 are shown fluorescence intensity profiles across the microchannel in the presence of various concentrations of hemoglobin. Inspection of these profiles leads to the conclusion that the detection limit of hemoglobin is $c a .10 \mu \mathrm{g} / \mathrm{mL}$.

The investigations described above have led to the development of a microfluidic sensor chip for the detection of hemoglobin based on a fluorescence quenching strategy. Thus, interaction of the readily prepared water-soluble conjugated polymer 3 with hemoglobin results in a concentration dependent decrease of fluorescence intensity in the microchannel. The strategy used to construct this system for rapid and sensitive 
detection of hemoglobin should be readily applied to create other conjugated polymer-based microfluidic sensor chips.

\section{Experimental Section}

Materials. The PPV precursor poly ( $p$-xylene tetrahydrothiophenium chloride) $\left(0.25 \mathrm{wt} \%\right.$ in $\left.\mathrm{H}_{2} \mathrm{O}\right)$ was purchased from Aldrich. Hemoglobin, bovine serum albumin (BSA), and chymotripsin were obtained from Sigma.

Preparation of the Microfluidic Chip. The microfluidic chip was fabricated with polydimethylsiloxane (PDMS) by using standard photolithography and molding techniques. First, a photoresist (PR) material (SU-8, Microchem.) was evenly spincoated at a thickness of $50 \mu \mathrm{m}$ on a 4-in Si wafer after cleaning with acetone and isopropyl alcohol (IPA) to fabricate a PDMS mold. The coated wafer was soft-baked at $65^{\circ} \mathrm{C}$ for $6 \mathrm{~min}$ and at $95{ }^{\circ} \mathrm{C}$ for $20 \mathrm{~min}$, cooled and irradiated with $365 \mathrm{~nm} \mathrm{UV}$ light using a UV exposer (MA6-II, SUSS Microtec) through a film-type photo mask with a patterned microchannel. A postexposure-baking of the wafer was performed at $65{ }^{\circ} \mathrm{C}$ for 1 min and at $95{ }^{\circ} \mathrm{C}$ for 5 min to enhance the crosslinking of PR. The wafer was developed for $6 \mathrm{~min}$ in a solution of propylene glycol monomethyl ether acetate (PGMEA). As a final process for the PDMS mold, the wafer was thoroughly washed with IPA to remove unexposed PR and was dried with a nitrogen gun. To fabricate the PDMS substrate, PDMS (DC-184A, Dow corning) was thoroughly mixed with a curing agent (DC-184B, Dow corning) in a $10: 1$ volume ratio and was degassed in a vacuum chamber for $30 \mathrm{~min}$. After being poured on to the mold, the PDMS prepolymer mixture was cured at $70^{\circ} \mathrm{C}$ for $2 \mathrm{~h}$, washed with IPA, exposed to ozone plasma (AH-1700, AHTECH LTS), and bonded to a glass slide.

Sensor Test. Fluorescent signals were imaged using a fluorescence microscope (BX71W, Olympus) with a 20x objective and a color CCD camera (DP70, Olympus). Two syringe pumps (KDS120, KD Scientic) were used to supply solutions of polymer and protein to the chip: one for the polymer flow and the other for the sheath flows of protein, maintaining the flow rates at $0.03 \mathrm{~mL} / \mathrm{h}$ and $0.01 \mathrm{~mL} / \mathrm{h}$. Syringes were connected to the inlets of the microchannels through capillaries and microfluidic fittings (LabSmith).

Acknowledgments. Financial support for this research was provided by Basic Science Research Program (NRF 200900
83161), and the Korea Research Foundation Grant (KRF-2006331-D00069) funded by the Korean Government (MOEHRD), and Seoul R\&D Program (10919).

\section{References}

1. Thomas III, S. W.; Joly, G. D.; Swager, T. M. Chem. Rev. 2007, $107,1339$.

2. Ahn, D. J.; Kim, J.-M. Acc. Chem. Res. 2008, 41, 805.

3. Ho, H.-A.; Najari, A.; Leclerc, M. Acc. Chem. Res. 2008, 41, 168.

4. Goldenberg, L. M.; Bryce, M. R.; Petty, M. C. J. Mater. Chem. 1999, 9, 1957.

5. Liu, B.; Bazan, G. C. Chem. Mater. 2004, 16, 4467.

6. Herland, A.; Inganäs, O. Macromol. Rapid Commun. 2007, 28, 1703.

7. Rose, A.; Zhu, Z.; Madigan, C. F.; Swager, T. M.; Bulović, V. Nature 2005, 434, 876.

8. Fan, C.; Plaxco, K. W.; Heeger, A. J. J. Am. Chem. Soc. 2002, 124, 5642.

9. Liu, B.; Bazan, G. C. J. Am. Chem. Soc. 2006, 128, 1188.

10. Kim, I.-B.; Bunz, U. H. F. J. Am. Chem. Soc. 2004, 126, 13685.

11. Tan, C.; Atas, E.; Müller, J. G.; Pinto, M. R.; Kleiman, V. D.; Schanze, K. S. J. Am. Chem. Soc. 2004, 126, 13685.

12. Lee, J. K.; Lee, T. S. J. Polym. Sci., Part A: Polym. Chem. 2005, 43, 1397.

13. Pun, C.-C.; Lee, K.; Kim, H.-J.; Kim, J. Macromolecules 2006, $39,7461$.

14. Charych, D. H.; Nagy, J. O.; Specak, W.; Bednarski, M. D. Science 1993, 261, 585.

15. Ma, G.; Müller, A. M.; Bardeen, C. J.; Cheng, Q. Adv. Mater. 2006, $18,55$.

16. Kolusheva, S.; Molt, O.; Herm, M.; Schrader, T.; Jelinek, R. J. Am. Chem. Soc. 2005, 127, 10000.

17. Yoon, J.; Chae, S. K.; Kim, J.-M. J. Am. Chem. Soc. 2007, 129, 3038.

18. Jang, J.; Ha, J.; Cho, J. Adv. Mater. 2007, 19, 1772.

19. Vilkner, T.; Janasek, D.; Manz, A. Anal. Chem. 2004, 76, 3373.

20. Song, G. H.; Heo, J.; Crooks, R. M. Anal. Chem. 2003, 75, 3161.

21. Kou, S.; Lee, H. N.; van Noort, D.; Swamy, K. M. K.; Kim, S. H.; Soh, J. H.; Lee, K.-M.; Nam, S.-W.; Yoon, J.; Park, S. Angew. Chem. Int. Ed. 2008, 47, 872.

22. Kim, P.; Kwon, K. W.; Park, M. C.; Lee, S. H.; Kim, S. M.; Suh, K. Y. Biochip J. 2008, $2,1$.

23. Eo, S.-H.; Song, S.; Yoon, B.; Kim, J.-M. Adv. Mater. 2008, 20, 1690.

24. Chen, X.; Lee, J.; Jou, M. J.; Kim, J.-M.; Yoon, J. Chem. Commun. 2009, 3434.

25. Pinto, M. R.; Kristal, B. M.; Schanze, K. S. Langmuir 2003, 19, 6523.

26. Kim, I.-B.; Dunkhorst, A.; Bunz, U. H. F. Langmuir 2005, 21, 7985. 